\title{
Characteristics associated with involuntary versus voluntary legal status at admission and discharge among psychiatric inpatients
}

Abstract Background The objective of this analysis was to determine the ways in which patients' legal statuses at hospital admission and discharge are associated with select sociodemographic and clinical variables. This study specifically investigated differences between patients who were voluntary during both admission and discharge, patients who were involuntary on admission but voluntary on discharge (having converted to voluntary status during hospitalization), and patients who were involuntary during both admission and discharge. Method Data were collected from the charts and treating clinicians of 227 consecutively discharged patients from two psychiatric units in a large, urban, county hospital in the southeastern United States. Based on results of bivariate tests, sociodemographic and clinical factors were entered into a polytomous logistic regression model to determine effect estimates (adjusted odds ratios). Results In the bivariate analyses, 15 variables were significantly associated with the trichotomous legal status. In the model, three factors were independently significantly associated with legal status, while controlling for four potential confounders: (1) whether or not the patient was experiencing psychotic symptoms at discharge, (2) whether or not the patient had documented medical problems requiring medication at discharge, and (3) the number of psychiatric medications. Conclusions A generalized lack of treatment engagement and adherence among involuntary patients likely underlies significant differences between the groups in terms of psychotic symptoms, diagnosed medical problems requiring medications, and

J. Craw $\cdot$ M.T. Compton MD, MPH $(\bowtie)$

Dept. of Psychiatry and Behavioral Sciences

Emory University School of Medicine

49 Jesse Hill Jr. Drive, S.E., Room \#333

Atlanta (GA) 30303, USA

E-Mail: mcompto@emory.edu number of psychiatric medications at discharge. Studying legal status (and the process of legal status conversion from involuntary to voluntary) and its correlates is an important topic for further research.

Key words commitment - compulsory - involuntary - voluntary

\section{Introduction}

In the United States, criteria for involuntary psychiatric hospitalization generally require imminent dangerousness to oneself or others, or complete inability to care for oneself $[1,2]$, though specific criteria vary between states [3]. An important rationale for involuntary psychiatric hospitalization is that patients admitted for evaluation often have severe deficits in their capacities to make treatment decisions $[4,5]$. Patients often revise their beliefs about the need for treatment during or after hospitalization. A study conducted by Gardner et al. [6] found that slightly more than half of patients who initially stated (upon admission) that they did not need hospitalization later admitted they indeed had needed treatment when reinterviewed several weeks after discharge.

It has been suggested that involuntary treatment may reduce the likelihood that patients will voluntarily seek care in the future [7, 8]. Subsequent aversion to psychiatric services may be due to a distrust of clinicians arising from coercive tactics or a lack of procedural justice (e.g., the patient is treated unfairly, disrespected, ignored, or excluded from the decision-making process) encountered during hospitalization $[1,8,9]$. On the other hand, involuntary hospitalization may provide patients with needed treatment, even if involuntarily, that results in improved outcomes and treatment adherence [10]. Some research indicates that the presence or absence of

䇺


coercion is accurately reflected by the patient's legal status, but other studies demonstrate that coercive pressures and perceived coercion do not necessarily correlate well with legal status in all settings $[8,11]$.

Evidence associating legal status with sociodemographic and clinical variables is sparse and inconsistent. One study found that higher re-admission rates and more unstable discharge living arrangements were associated with involuntary legal status on hospital admission [12]. A meta-analytic review conducted using studies from the 1950s through the early 1980s found that the only significant outcome measure correlated with admission legal status was length of stay [13]. Studies have reached opposing conclusions regarding whether or not male gender is significantly associated with involuntary legal status, especially when controlling for severity of illness $[7,12,14]$. With regard to clinical characteristics, psychotic disorder diagnoses, greater illness severity, and more frequent preadmission assaultive behaviors may be associated with involuntary admission [13]. Even fewer studies have examined factors associated with converting from involuntary to voluntary legal status during hospitalization [14-16]. Cuffel [14] identified greater clinical improvement, less severe diagnoses, and nonminority ethnicity as factors predictive of converting to voluntary status early in the hospitalization. Later during hospitalization, the only factor associated with legal status conversion was having discharge living arrangements involving family and friends.

Studies that find significant associations between legal status and outcomes may influence policies relating to resource allocation during hospitalization, discharge planning, and outpatient follow-up [12, 15]. For instance, research characterizing those at risk for very short involuntary hospitalization (i.e., discharge upon expiration of short-term commitment) addresses the need for more thorough and engaged evaluation and treatment while hospitalized. One may be able to enhance treatment adherence with a more focused treatment and evaluation plan, in conjunction with closer follow-up after discharge [12]. Though legal status is often overlooked in studies in which it would be particularly relevant, it is clearly an important variable for further research.

The purpose of the present study was to compare three groups of hospitalized psychiatric patients: (1) patients admitted and discharged voluntarily (VOLVOL), (2) patients admitted involuntarily who later signed-in voluntarily and were discharged voluntarily (INV-VOL), and (3) patients admitted and discharged on an involuntary legal status (INV-INV). As such, this trichotomous criterion variable addressed the change (or lack thereof) in patients' legal status during hospitalization, allowing an examination of whether or not certain sociodemographic and clinical factors are associated with differences in admissionto-discharge legal status. Treating legal status as a trichotomous variable (rather than solely considering either admission or discharge legal status) may yield meaningful results that add to the current body of research on correlates of patients' legal status.

\section{Methods}

\section{Subjects and setting}

This analysis was part of a study aimed at determining predictors of non-adherence with the first scheduled community mental health outpatient appointment following hospitalization [17]. Inclusion in the study was restricted to consecutively discharged patients with a scheduled follow-up appointment at any of 12 participating community mental health settings in a single urban county. Patients rehospitalized during the course of data collection were not included a second time, in order to maintain independence of observations. This analysis included data that were collected on 227 patients from December 2003 to July 2004.

All patients were admitted to the 22-bed inpatient unit or the 8bed crisis stabilization unit of a large, public-sector hospital in the southeastern United States. Both the longer-stay inpatient unit and the crisis stabilization unit admit patients primarily for evaluation and treatment of first episodes of illness or exacerbations of severe and persistent mental illnesses (primarily schizophrenia and other psychotic disorders and severe affective disorders). This county hospital serves a predominantly low-income, urban, African American population.

In the state of Georgia, commitment for involuntary evaluation can be initiated by a licensed physician, psychologist, clinical social worker, or psychiatric clinical nurse specialist. Taking custody for transportation to an emergency evaluation facility also can be carried out by a law enforcement officer. In either case, this initial commitment allows for the individual to be taken to and held at a locked evaluation facility. Commitment criteria include the fact that the person appears to have a mental illness, and either (1) presents a substantial risk of imminent harm to self or others as manifested by recent overt acts or threats of violence presenting a probability of physical injury, or (2) is so unable to care for his/her own physical health and safety as to create an imminently life-endangering crisis [3]. Within $48 \mathrm{~h}$ of admission, if the individual continues to meet commitment criteria, a physician may sign for commitment for further evaluation and treatment of up to five business days in duration. During this period of time, some patients will sign-in as voluntary patients. If the patient does not signin voluntarily, then he or she is typically discharged after the 5 days, unless commitment criteria clearly continue to be present. In that case, a court hearing is scheduled to determine whether or not the legal standard is met for longer-term involuntary hospitalization. However, in this setting extended commitment is used relatively rarely, and only in circumstances in which the threat to self or others is very apparent.

\section{Procedures}

The majority of data collected were abstracted from patients' medical charts. Subjectively rated variables (e.g., treatment plan adherence during hospitalization, the treatment team's opinion of likelihood of follow-up) were obtained by consulting with the social worker, the psychiatry resident or psychology intern, and the attending psychiatrist caring for the patient during hospitalization. As described in two recently published reports, basic demographic, socioeconomic, psychosocial, and clinical data were recorded in a systematic fashion using a standardized data collection instrument $[17,18]$. The criterion variable of interest in this analysis was a three-level variable representing the patient's legal status at admission and at discharge: VOL-VOL, INV-VOL, and INV-INV. The research was approved by the university's institutional review board and the hospital's research oversight committee. 
Table 1 Basic sociodemographic and clinical descriptive statistics for the overall sample $(n=227)$

\begin{tabular}{lcr}
\hline Variable & Frequency & \\
\hline Gender, female & $126(55.5 \%)$ & \\
Race & $191(84.9 \%)$ & \\
$\quad$ Black/African American & $4(1.8 \%)$ & \\
$\quad$ Hispanic/Latino & $28(12.4 \%)$ & \\
$\quad$ White/Caucasian/European American & $2(0.9 \%)$ & \\
$\quad$ Other & $213(94.2 \%)$ & \\
Employment status, unemployed & $58(25.6 \%)$ & \\
Homeless on admission & $154(67.8 \%)$ & Range \\
Marital status & $19(8.4 \%)$ & $17-76$ \\
$\quad$ Single/never married & $54(23.8 \%)$ & \\
$\quad$ Married/living as married & $169(75.1 \%)$ & \\
$\quad$ Separated/divorced/widowed & $194(85.8 \%)$ & 18 \\
Prior psychiatric hospitalization & Mean \pm SD & \\
Psychotic symptoms present on admission & $38.7 \pm 12.4$ & 12 \\
\hline Variable & $12.1 \pm 2.3$ & 30 \\
\hline Age & $30.3 \pm 8.2$ & \\
Years of education & & \\
GAF score on admission & & \\
\hline
\end{tabular}

\section{Analysis}

Bivariate analyses were performed using a variety of independent variables in relation to the criterion variable. Continuous variables were analyzed using one-way analysis of variance and categorical variables were analyzed using chi-square tests of association. These bivariate analyses were conducted to inform the selection of variables for inclusion in the subsequent multivariate regression analysis.

Polytomous logistic regression modeling was required due to the trichotomous nature of the admission/discharge legal status criterion variable. Those patients admitted and discharged voluntarily (VOL-VOL) were designated as the referent group in the regression model. Entering variables into the logistic model was based on significant bivariate test results $(P<0.05)$. A thorough assessment of confounding was conducted, and relevant confounding variables were controlled for in the model. The backward elimination method was used to identify variables that were independently significantly associated $(P<0.05)$ with legal status. Appropriate modeling diagnostics were conducted to ensure the fit of the model to the data. Adjusted odds ratios and 95\% confidence intervals were computed to determine effect estimates for independent variables that were significantly associated with legal status, controlling for all other covariates in the model. The SAS version 9.1 software (SAS Inc., Cary, North Carolina, USA) was used for all statistical analyses.

\section{Results}

Basic sociodemographic and clinical descriptive statistics for the overall sample are shown in Table 1. Slightly over half $(55.5 \%)$ of patients were female. The racial composition of the sample is consistent with that of the population served by the two inpatient units, and by the hospital in general. Specifically, $84.9 \%$ of patients were Black/African American. At the time of admission, $94.2 \%$ of patients were unemployed, and $25.6 \%$ reported being homeless. The majority of patients were single/never married $(67.8 \%)$. The majority of patients had been previously hospitalized for psychiatric treatment $(75.1 \%)$. An even greater percentage of patients were reported as having psychotic symptoms at the time of admission
$(85.8 \%)$. The mean age was $38.7 \pm 12.4$ years, and the mean educational level was $12.1 \pm 2.3$ years. The mean Global Assessment of Functioning (GAF) scale score on admission was $30.3 \pm 8.2$, ranging from 10 to 55.

Among the 227 patients, $56(24.7 \%)$ were in the VOL-VOL group, $81(35.7 \%)$ were in the INV-VOL group, and $90(39.6 \%)$ were in the INV-INV group. As shown in Table 2, select demographic and social variables were examined across the three groups. The difference in percentage of patients documented as having Axis IV psychosocial problems in the area of housing was statistically significant $\left(\chi^{2}=6.43, \mathrm{df}=2\right.$, $P=0.04)$, with patients admitted and discharged voluntarily most likely to have housing problems $(60.7 \%)$. The percentage of patients who were receiving disability payments-Supplemental Security Income (SSI) or Social Security Disability Insurance (SSDI) - over the past 3 months also differed significantly across the three legal status groups $\left(\chi^{2}=6.82\right.$, $\mathrm{df}=2, P=0.03)$, with patients admitted and discharged involuntarily most likely to be receiving disability payments $(61.1 \%)$.

Table 3 shows select clinical and diagnostic characteristics. The three legal status groups differed significantly on 13 of these 20 variables. The INV-INV group was most likely to have had previous psychiatric hospitalizations (84.3\%), followed by $71.6 \%$ among the INV-VOL group, and $65.5 \%$ among the VOL-VOL group $\left(\chi^{2}=7.27, \mathrm{df}=2, P=0.03\right)$. Compared to the VOL-VOL group, patients who were admitted and discharged involuntarily were more likely to have a primary diagnosis of a psychotic disorder, to have a change in GAF score from admission to discharge of $<20$ points, and to have psychotic symptoms on discharge. Patients admitted and discharged involuntarily were less likely to have documentation of medical problems requiring medications at discharge, to be taking $>2$ psychiatric 
Table 2 Sociodemographic characteristics of 227 hospitalized patients

\begin{tabular}{|c|c|c|c|c|c|}
\hline \multirow[b]{2}{*}{ Variable } & \multicolumn{3}{|c|}{ Legal status on admission and discharge ${ }^{a}$} & \multirow[b]{2}{*}{$\begin{array}{l}\text { Test statistic } \\
\text { (df) }\end{array}$} & \multirow[b]{2}{*}{$P$-value } \\
\hline & $\begin{array}{l}\text { VOL-VOL } \\
(n=56)\end{array}$ & $\begin{array}{l}\text { INV-VOL } \\
(n=81)\end{array}$ & $\begin{array}{l}\text { INV-INV } \\
(n=90)\end{array}$ & & \\
\hline Gender, female & $32(57.1 \%)$ & $48(59.3 \%)$ & $46(51.1 \%)$ & $1.23(2)$ & 0.54 \\
\hline Race, Black/African American & $45(81.8 \%)$ & $66(81.5 \%)$ & $80(89.9 \%)$ & $2.87(2)$ & 0.24 \\
\hline Employed part-time or full-time & $2(3.64 \%)$ & 7 (8.64\%) & $4(4.44 \%)$ & $1.99(2)$ & 0.37 \\
\hline Homeless on admission & $17(30.4 \%)$ & $23(28.4 \%)$ & $18(20.0 \%)$ & $2.48(2)$ & 0.29 \\
\hline \multicolumn{6}{|l|}{ Current marital } \\
\hline Single/never married & $32(57.1 \%)$ & 56 (69.1\%) & $66(73.3 \%)$ & & \\
\hline Married/living as married & $6(10.7 \%)$ & $7(8.6 \%)$ & $6(6.7 \%)$ & $4.32(4)$ & 0.36 \\
\hline Separated/divorced/widowed & $18(32.1 \%)$ & $18(22.2 \%)$ & $18(20.0 \%)$ & & \\
\hline Age on admission (Mean $\pm S D$ ) & $41.0 \pm 11.5$ & $37.0 \pm 13.2$ & $38.8 \pm 12.1$ & $1.73(222)$ & 0.18 \\
\hline Axis IV housing problems & $34(60.7 \%)$ & $35(43.2 \%)$ & $36(40.0 \%)$ & $6.43(2)$ & 0.04 \\
\hline Axis IV economic problems & $32(57.1 \%)$ & $51(63.0 \%)$ & $41(45.6 \%)$ & $5.40(2)$ & 0.07 \\
\hline $\begin{array}{l}\text { Receiving disability payments over } \\
\text { past } 3 \text { months (SSI or SSDI) }\end{array}$ & $22(39.3 \%)$ & $40(49.4 \%)$ & $55(61.1 \%)$ & $6.82(2)$ & 0.03 \\
\hline
\end{tabular}

aLegal status on admission and discharge: VOL-VOL, Patients having voluntary legal status on both admission and discharge; INV-VOL, Patients having involuntary status on admission and voluntary status on discharge; INV-INV, Patients having involuntary legal status on both admission and discharge medications at discharge, and to have good treatment plan adherence during hospitalization compared to the VOL-VOL group. Not unexpectedly, length of stay in days $(F=20.45, \mathrm{df}=224, P<0.0001)$ and the inpatient unit to which patients were admitted $\left(\chi^{2}=32.84, \mathrm{df}=2, P<0.0001\right)$ were highly significantly different across the groups.

The average lengths of hospital stay (in days) across the three groups of patients are displayed in Fig. 1. Patients in the INV-INV group had an average length of hospitalization of 8.4 days, whereas patients in the VOL-VOL group had an average length of stay of 11.3 days. Patients in the INV-VOL group had the longest average length of hospital stay, at 15.5 days. Based on post-hoc tests using the Tukey approach, the difference between mean lengths of stay was significant for the INV-VOL group compared to the VOL-VOL group and for the INV-VOL group compared to the INV-INV group.

Figure 2 illustrates the differences among the three groups in terms of the percentage of patients adhering with the first scheduled community mental health

Table 3 Clinical and diagnostic characteristics of 227 hospitalized patients

\begin{tabular}{|c|c|c|c|c|c|}
\hline \multirow[b]{2}{*}{ Variable } & \multicolumn{3}{|c|}{ Legal status on admission and discharge ${ }^{a}$} & \multirow[b]{2}{*}{$\begin{array}{l}\text { Test statistic } \\
\text { (df) }\end{array}$} & \multirow[b]{2}{*}{$P$-value } \\
\hline & $\begin{array}{l}\text { VOL-VOL } \\
(n=56)\end{array}$ & $\begin{array}{l}\text { INV-VOL } \\
(n=81)\end{array}$ & $\begin{array}{l}\text { INV-INV } \\
(n=90)\end{array}$ & & \\
\hline Prior psychiatric hospitalization & $36(65.5 \%)$ & $58(71.6 \%)$ & 75 (84.3\%) & $7.27(2)$ & 0.03 \\
\hline Psychotic symptoms on admission & 43 (76.8\%) & 71 (87.7\%) & $80(89.9 \%)$ & $5.20(2)$ & 0.07 \\
\hline Primary diagnosis, schizophrenia or other psychotic disorder & $26(47.3 \%)$ & 56 (69.1\%) & $63(70.0 \%)$ & $9.03(2)$ & 0.01 \\
\hline Comorbid substance use disorder diagnosis & $26(46.4 \%)$ & $34(42.5 \%)$ & 40 (44.4\%) & $0.21(2)$ & 0.90 \\
\hline Comorbid personality disorder diagnosis & $10(17.9 \%)$ & $13(16.1 \%)$ & $12(13.3 \%)$ & $0.58(2)$ & 0.75 \\
\hline Change of $<20$ in GAF score (Discharge-Admission) & $23(42.6 \%)$ & $26(32.9 \%)$ & $47(53.4 \%)$ & $7.14(2)$ & 0.03 \\
\hline Depressive symptoms at discharge & $23(41.1 \%)$ & $23(28.4 \%)$ & $22(24.4 \%)$ & $4.69(2)$ & 0.10 \\
\hline Anxious symptoms at discharge & $23(41.1 \%)$ & 43 (51.1\%) & $28(31.1 \%)$ & $8.49(2)$ & 0.01 \\
\hline Psychotic symptoms at discharge & $27(48.2 \%)$ & $51(63.0 \%)$ & $67(74.4 \%)$ & $10.34(2)$ & $<0.01$ \\
\hline Documented medical problems requiring medication at discharge & $35(62.5 \%)$ & $41(50.6 \%)$ & $32(35.6 \%)$ & $10.52(2)$ & $<0.01$ \\
\hline$>2$ Psychiatric medications at discharge & $20(36.4 \%)$ & $13(16.1 \%)$ & $14(15.9 \%)$ & $10.40(2)$ & $<0.01$ \\
\hline Experiencing side effects at discharge & $7(12.5 \%)$ & $23(28.4 \%)$ & $13(14.4 \%)$ & $7.41(2)$ & 0.02 \\
\hline Required seclusion & $5(8.9 \%)$ & $11(13.6 \%)$ & $18(20.0 \%)$ & $3.52(2)$ & 0.17 \\
\hline Required restraints & $4(7.1 \%)$ & $8(9.9 \%)$ & $8(8.9 \%)$ & $0.31(2)$ & 0.86 \\
\hline Required PRN medicine & $14(25.0 \%)$ & $30(37.0 \%)$ & $38(42.2 \%)$ & $4.48(2)$ & 0.11 \\
\hline Patient has an established outpatient clinician & $15(26.8 \%)$ & $20(25.0 \%)$ & $38(42.2 \%)$ & $6.78(2)$ & 0.03 \\
\hline & \multicolumn{5}{|c|}{ Treatment team's opinion of likelihood of follow-up } \\
\hline Poor & $15(26.8 \%)$ & $16(20.0 \%)$ & $35(38.9 \%)$ & $15.02(4)$ & $<0.01$ \\
\hline Fair & $19(34.0 \%)$ & $29(36.3 \%)$ & $38(42.2 \%)$ & & \\
\hline Good & $22(39.3 \%)$ & $35(43.8 \%)$ & $17(18.9 \%)$ & & \\
\hline Length of stay in days (Mean \pm SD) & $11.3 \pm 7.5$ & $15.5 \pm 8.4$ & $8.4 \pm 6.0$ & $20.45(224)$ & $<0.0001$ \\
\hline Unit, longer-stay inpatient unit & $36(64.3 \%)$ & $67(83.8 \%)$ & $37(41.1 \%)$ & $32.84(2)$ & $<0.0001$ \\
\hline
\end{tabular}

${ }^{a}$ Legal status on admission and discharge: VOL-VOL, Patients having voluntary legal status on both admission and discharge; INV-VOL, Patients having involuntary status on admission and voluntary status on discharge; INV-INV, Patients having involuntary legal status on both admission and discharge 


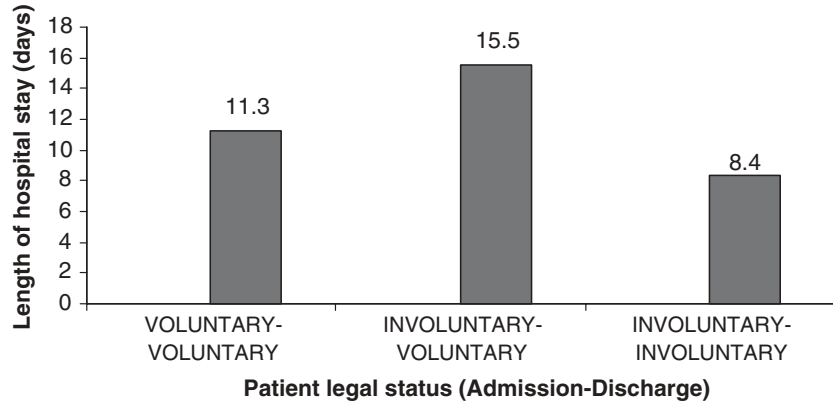

Fig. 1 Average lengths of hospital stay (days) in the three groups of patients

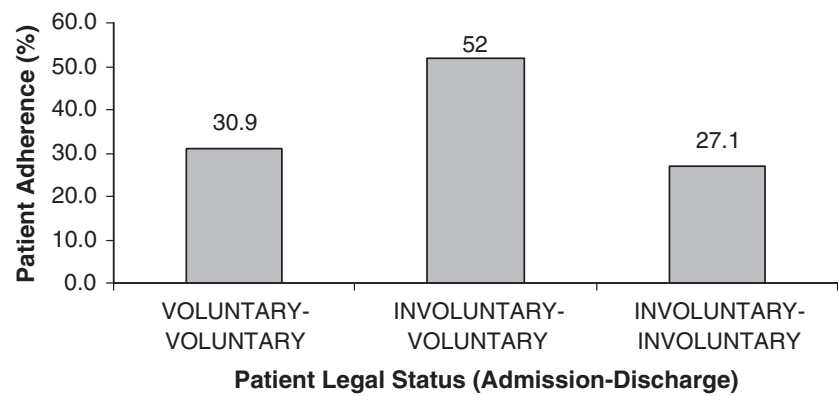

Fig. 2 Percentage of patients adhering with the first scheduled community mental health appointment following hospitalization

appointment following hospitalization. The average time interval between hospital discharge and the first scheduled community mental health appointment was 14 days and ranged from 0 (i.e., the appointment was scheduled to occur on same day as discharge) to 59 days. (Follow-up was assessed by a single phone call to the community mental health center where the discharged patient had been scheduled for the first outpatient appointment. The follow-up phone call was made a few days after the appointment, and further outpatient adherence was not tracked [17].) Patients in the INV-VOL group were the most likely to adhere with the first follow-up appointment $(52.0 \%)$, whereas patients in the VOL-VOL group (30.9\%) and the INVINV group $(27.1 \%)$ were far less likely to adhere $\left(\chi^{2}=11.75, \mathrm{df}=2, P<0.01\right)$.

Based on the significant bivariate test results and a thorough consideration of relevant confounding variables, 18 factors were entered into the polytomous logistic regression model. In this model, three of these factors were independently associated with legal status: psychotic symptoms at discharge, documented medical problems requiring medication, and number of psychiatric medications. Psychiatric inpatient unit, length of hospital stay, prior psychiatric hospitalization, and receiving disability payments (SSI/SSDI) were deemed confounding variables and were, therefore, controlled for in the final model. Because of sparse missing values, modeling results were generated using data from 221 individuals. Table 4 shows model coefficients, standard errors, adjusted odds ratios, and $95 \%$ confidence intervals for each of the three significant variables, by legal status category.

Patients who were involuntary on admission and discharge (INV-INV) were approximately 4.4 times more likely to have psychotic symptoms at discharge, relative to the VOL-VOL group. Patients in the INVINV group were also about 4.5 times less likely (or equivalently, 0.22 times as likely) to have documented medical problems requiring medications at discharge, relative to those in the VOL-VOL group. Patients who were involuntary on admission and voluntary on discharge (INV-VOL) were approximately 6 times less likely ( 0.17 times as likely) to be prescribed more than two psychiatric medications at discharge.

\section{Discussion}

Multivariate logistic regression modeling yielded three clinical variables that were independently significantly associated with admission/discharge legal status, even after controlling for psychiatric unit, length of hospital stay, prior psychiatric hospitalization, and whether or not the patient received disability payments. As described below, the associations between legal status and each of these three significant variables potentially may be explained by the lower overall treatment engagement common among involuntary patients.

First, patients in the INV-INV group were much more likely to be experiencing psychotic symptoms at discharge, compared to the VOL-VOL group. The
Table 4 Independent variables significantly associated with admission and discharge legal status among 221 hospitalized patients

\begin{tabular}{llrrrr}
\hline Variable & Legal status category $^{\mathrm{a}}$ & \multicolumn{1}{l}{$\mathrm{B}$} & $\mathrm{SE}$ & $\mathrm{aOR}$ & $95 \% \mathrm{Cl}$ \\
\hline Psychotic symptoms at discharge & INV-VOL & -0.02 & 0.44 & 0.98 & $0.42,2.32$ \\
& INV-INV & 1.49 & 0.46 & 4.42 & $1.80,10.86$ \\
& INV-VOL & -0.48 & 0.40 & 0.62 & $0.28,1.35$ \\
Documented medical problems & -1.51 & 0.43 & 0.22 & $0.10,0.51$ \\
requiring medication at discharge & INV-INV & -1.80 & 0.52 & 0.17 & $0.06,0.46$ \\
Number of psychiatric medications & INV-VOL & -0.88 & 0.51 & 0.41 & $0.15,1.12$ \\
at discharge $(>2)$ & INV-INV & & \\
\hline
\end{tabular}

The model controls for psychiatric hospital unit, length of hospital stay, prior psychiatric hospitalization, and receiving disability payments (SSI/SSDI)

${ }^{a}$ VOL-VOL (voluntary legal status on both admission and discharge) is the referent group for all comparisons; INV-VOL, involuntary legal status on admission, voluntary status on discharge; INV-INV, involuntary legal status on both admission and discharge

$\mathrm{B}$, Coefficient value from the final regression model; SE, standard error; aOR, adjusted odds ratio; $\mathrm{Cl}$, confidence interval 
most plausible explanation for this finding relates to a difference in the level of acceptance of treatment between the two groups. Involuntary legal status is generally an indicator of a lack of treatment engagement, resistance to treatment, and/or impaired insight. Bivariate test results for "treatment plan adherence during the week prior to discharge" support the idea that involuntary patients generally had poorer overall treatment adherence.

When patients refuse to sign-in voluntarily and no longer meet dangerousness criteria, they may have to leave the hospital before resolution of psychotic symptoms. The abbreviated hospitalization may not have allowed for sufficient time for stabilization and adjustments to psychiatric medications. Due to the laws governing involuntary inpatient commitment in this state, individuals who have been hospitalized for the duration of the short-term commitment and who have refused to convert to voluntary status, must be discharged unless they clearly meet further commitment criteria [3]. On the other hand, patients with voluntary legal status are generally discharged based on clinical, rather than legal, criteria-they are released from the hospital when symptoms have sufficiently resolved and the patient is stable enough to return to the community.

A significant difference in psychotic symptoms at discharge was not found between the INV-VOL group and the VOL-VOL group. This is not surprising given the fact that these two groups were virtually equally as likely to be rated as having good treatment adherence during the week prior to discharge. Furthermore, these patients were discharged after sufficient resolution of symptoms rather than legal expiration. From a clinical vantage point, it appears that INV-INV patients are being discharged "too soon" due to legal constraints.

An alternate explanation for the difference in psychotic symptoms at discharge among the three groups is that INV-INV patients were more likely to have psychotic symptoms at the time of admission compared to the other two groups. However, the bivariate test revealed no statistically significant difference between the three groups in terms of psychotic symptoms present on admission.

Second, patients admitted and discharged involuntarily (INV-INV) were much less likely to have documented medical problems requiring medications at the time of discharge, relative to the VOL-VOL group. Because length of stay was controlled for in the model, the most likely reason for the difference in medical problems at discharge is a generalized lack of treatment engagement among involuntary patients. That is, poor treatment engagement is associated with a decreased likelihood of medical problems being reported and diagnosed. Involuntary patients are more likely to refuse medical history taking, physical examination, vital sign checks, diagnostic blood work, and other diagnostic procedures during hospitaliza- tion. Thus, clinicians may not have sufficient opportunities or information to detect common medical problems, such as hypertension, diabetes mellitus, and anemia.

It is notable that a significant difference in documented medical problems requiring medication was not found between the INV-VOL and VOL-VOL groups. As noted earlier, these two groups had similar treatment adherence during the week prior to discharge. These results support the idea that converting to voluntary status is associated with better treatment engagement, affording more opportunities for medical problems to be detected and medication to be prescribed. Future research is needed to determine whether a general lack of treatment engagement alone, or some additional factors in combination with poor treatment engagement, accounts for the difference in documented medical problems requiring medication.

Some alternate explanations as to why the INVINV patients had fewer medical problems requiring medication at discharge merit consideration. For example, it is conceivable that even when clinicians detect a medical problem, they may be biased toward not prescribing medication to involuntary patients who are highly unlikely to agree to take it. However, in this particular study setting, when medical problems are detected in involuntary patients, clinicians typically prescribe the indicated medication, regardless of the likelihood that the patient will comply with taking it. Another possible explanation is that patients in the INV-INV group are generally healthier than the other two groups. However, because the three legal status groups were very similar with respect to age, gender, and race, it is highly unlikely that one group of patients would have been healthier than the other two.

Third, though the INV-VOL and VOL-VOL groups did not differ with respect to psychotic symptoms and medical problems requiring medication at discharge, these two groups did differ in the number of psychiatric medications at time of discharge. Those patients in the INV-VOL group were much less likely to have been prescribed more than two psychiatric medications at discharge, relative to the VOL-VOL group. The difference in number of psychiatric medications between the INV-INV and VOL-VOL groups was not significant, though the effect for the INV-INV group was in the same direction as with the INV-VOL group. The most plausible explanation for this finding may be related to both a lower overall treatment engagement among involuntary patients and differences in clinician prescribing behavior for involuntarily admitted patients compared to voluntary patients. That is, perhaps clinicians err on the side of caution with patients recently converting to voluntary status by limiting the number of psychiatric medications used to treat symptoms. Clinicians may limit the number of prescribed medications, especially if psy- 
chiatric symptoms are resolving, in order to simplify the medication regimen and sustain good treatment adherence. Interestingly, a much higher percentage of patients in the INV-VOL group adhered with the first community mental health appointment compared to the VOL-VOL group and the INV-INV group. It is conceivable that a simpler treatment regimen (one or two psychiatric medications) may contribute to higher rates of adherence with initial community mental health follow-up.

Several methodological limitations of this study merit consideration. First, the fact that patients are admitted voluntarily or involuntarily is a legal distinction, which may not accurately reflect the patient's level of treatment engagement $[11,12,19,20]$. Patients admitted voluntarily do not necessarily always have the motivation and adherence behaviors of individuals truly pursuing treatment for mental illness of their own accord [11-13]. For example, a voluntary patient who is pressured or coerced by family members to seek inpatient treatment may have motivation, adherence, and insight more similar to patients admitted involuntarily than to those voluntarily seeking treatment $[11,12]$. Likewise, some involuntary patients may be more similar to voluntary patients than to other involuntary patients. This might occur when patients who would willingly have signed-in voluntarily are admitted involuntarily in order to overcome obstacles associated with voluntary admission procedures in certain mental health systems [11]. Because legal status may not be an appropriate surrogate for measuring perceived coercion or treatment engagement $[5,8,11]$, future research should consider utilizing MacArthur's Perceived Coercion Scale [21] and a continuous scale to assess treatment engagement.

A second methodological limitation is that commitment statutes "censor" the number of patients that convert to voluntary status, since non-converters must be discharged upon expiration of the commitment. Therefore, the true difference between the INVVOL and INV-INV groups is somewhat obscured because of the limited timeframe in which patients have to convert to voluntary status. One cannot assume that any given INV-INV patient would never have converted to voluntary status; rather one can only infer that he or she had not done so by time of discharge. In other words, caution must be exercised in assigning ontological status to the INV-INV group. A third limitation is that patients re-admitted during the data collection period were excluded from the analysis. Future research might consider focusing on INV-INV patients with multiple hospitalizations. Another limitation with regard to data collection was that clinical and diagnostic variables (some of which were subjectively rated) were based on clinicians' reports or information in the hospital chart, without using rating scales administered directly to patients. However, given the goals of this study, the researchers deemed patient assessments, beyond that done as part of routine evaluation and treatment, unnecessary. Also, involuntary patients would have been less likely to participate in formal research evaluations compared to voluntary patients, thus restricting the ability of the analyses to examine the issues of interest. Lastly, the findings from this study may have limited generalizability, given the specific sociodemographic and diagnostic characteristics of the sample. However, the population of interest to the researchers was that of minority individuals with severe psychiatric illnesses being treated in a large, urban, public-sector hospital.

Despite the methodological limitations inherent in the study design, there were also several strengths in the methodology and data analysis process. First, an extremely thorough assessment of confounding was conducted. The final model controlled for four variables that were considered likely confounders. Second, alternate modeling techniques were conducted, all of which resulted in the same findings. Lastly, a concerted effort was made to minimize missing values during data collection, and to prevent biases, the few variables with significant missing values were excluded from the analysis.

\section{Conclusions}

Research on associations between patients' legal status and sociodemographic and clinical variables can directly and indirectly affect psychiatric treatment during and after hospitalization. For instance, patients in the INV-INV group have the shortest average length of hospitalization, which is a direct result of the commitment law dictating that patients cannot be involuntarily hospitalized for more than 5 days unless they continue to meet dangerousness criteria [3], even if symptoms have not yet resolved. In addition to being more likely to have psychotic symptoms at discharge, patients in the INV-INV group are less likely to have documented medical problems requiring medication at discharge. Thus, involuntary patients frequently may be discharged from the hospital with unresolved psychotic symptoms and undetected, untreated medical problems. The poor outpatient treatment followup rate among INV-INV patients suggests that this is a particularly vulnerable group needing focused attention by the mental health system.

During hospitalization, clinicians should be attentive to the manner in which involuntary patients are treated because this can greatly influence patients' perceptions of coercion. It has been suggested that a concerted effort to reduce the level of coercive practices used during the admission process could mitigate some of the negative effects of coercion, such as future non-adherence with treatment and outpatient follow-up [8]. After discharge from the hospital, involuntary outpatient commitment can be employed 
to stabilize patients in the community, though this form of coercive treatment is also controversial. Recent research has suggested efficacy of involuntary outpatient commitment in enhancing treatment compliance and reducing re-admission rates among persons with severe psychiatric illnesses $[22,23]$. Increased resource allocation for involuntary patients during the admission process and throughout hospitalization, discharge planning, and outpatient followup may have the downstream effect of preventing future hospital admissions and worsening of medical comorbidity.

\section{References}

1. Kaltiala-Heino R, Laippala P, Salokangas RKR (1997) Impact of coercion on treatment. Int J Law Psychiatry 20(3):311-322

2. Fisher WH, Barreira PJ, Lincoln AK, Simon LJ, White AW, RoyBujnowski K, Sudders M (2001) Insurance status and length of stay for involuntarily hospitalized patients. J Behav Health Serv Res 28(3):334-346

3. Georgia legal code. Sections: 37-3-41, 37-3-42, 37-3-43. Web access at http://www.legis.state.ga.us/legis/GaCode

4. Monahan J, Swartz M, Bonnie RJ (2003) Mandated treatment in the community for people with mental disorders. Health Affairs 22(5):28-35

5. Lidz CW, Mulvey EP, Hoge SK, Kirsch BL, Monahan J, Eisenberg M, Gardner W, Roth LH (1998) Factual sources of psychiatric patients' perceptions of coercion in the hospital admission process. Am J Psychiatry 155(9):1254-1260

6. Gardner W, Lidz CW, Hoge SK, Monahan J, Eisenberg MM, Bennett NS, Mulvey EP, Roth LH (1999) Patients' revisions of their beliefs about the need for hospitalization. Am J Psychiatry 156(9):1385-1391

7. Lindsey K, Paul GL (1989) Involuntary commitments to public mental institutions: issues involving the over-representation of blacks and assessment of relevant functioning. Psychol Bull 106:171-183

8. Lidz CW, Hoge SK, Gardner W, Bennett NS, Monahan J, Mulvey EP, Roth LH (1995) Perceived coercion in mental hospital admission: pressures and process. Arch Gen Psychiatry 52(12):1034-1039
9. Swartz MS, Swanson JW, Hannon MJ (2003) Does fear of coercion keep people away from mental health treatment? Evidence from a survey of persons with schizophrenia and mental health professionals. Behav Sci Law 21:459-472

10. Grudzinskas AJ (2002) Commentary: the search for a formula to relate competence, coercion, and mandated treatment. J Am Acad Psychiatry Law 30(2):218-220

11. Hoge SK, Lidz CW, Eisenberg M, Gardner W, Monahan J, Mulvey E, Roth L, Bennett N (1997) Perceptions of coercion in the admission of voluntary and involuntary psychiatric patients. Int J Law Psychiatry 20(2):167-181

12. Houston KG, Mariotto M (2001) Outcomes for psychiatric patients following first admission: relationships with voluntary and involuntary treatment and ethnicity. Psychol Rep 88:10121014

13. Nicholson RA (1986) Correlates of commitment in psychiatric patients. Psychol Bull 100:241-250

14. Cuffel BJ (1992) Characteristics associated with legal status change among psychiatric inpatients. Commun Mental Health J 28(6):471-482

15. Anand VS, Ciccone JR, Kashtan I, Seger AS (2002) Factors predictive of changes in the legal status of psychiatric inpatients. J Forensic Sci 47(6):1365-1369

16. Nicholson RA (1988) Characteristics associated with change in the legal status of involuntary psychiatric patients. Hospital Commun Psychiatry 39:424-429

17. Compton MT, Rudisch BE, Craw J, Thompson T, Owens DA (2006) Predictors of missed first appointments at community mental health centers after psychiatric hospitalization. Psychiatr Serv 57(4):531-537

18. Compton MT, Craw J, Rudisch BE (2006) Determinants of inpatient psychiatric length of stay in an urban county hospital. Psychiatr Quart 77(2):173-188

19. Okin RL (1986) The relationship between legal status and patient characteristics in state hospitals. Am J Psychiatry 143:1233-1237

20. Hoge SK, Lidz CW, Eisenberg M, Monahan J, Bennett N, Gardner W, Mulvey E, Roth L (1998) Family, clinician, and patient perceptions of coercion in mental hospital admission: a comparative study. Int J Law Psychiatry 21(2):131-146

21. Gardner W, Hoge SK, Bennett N, Roth LH, Lidz CW, Monahan J, Mulvey EP (1993) Two scales for measuring coercion during mental hospital admission. Behav Sci Law 11:307-322

22. Torrey EF, Zdanowicz M (2001) Outpatient commitment: what, why, and for whom. Psychiatr Serv 52(3):337-341

23. Appelbaum PS (2001) Thinking carefully about outpatient commitment. Psychiatr Serv 52(3):347-350 